\title{
Naturalização da Fenomenologia e perspectivas metodológicas na pesquisa experimental em Psicologia
}

\author{
Phenomenology Naturalization and methodological \\ perspectives on Psychology \\ experimental research
}

Thiago Gomes de CASTRO'

William Barbosa GOMES ${ }^{2}$

\section{Resumo}

O artigo explora a relação entre Fenomenologia e Ciências Cognitivas a partir da literatura internacional denominada naturalização da fenomenologia. São discutidas as tendências de pesquisa na área. O estudo contrasta a pesquisa com métodos introspectivos da metodologia básica em Ciências Cognitivas e Fenomenologia Experimental. Nas tendências de naturalização da Fenomenologia, destaca-se a Fenomenologia front-loaded, caracterizada como um tipo de fenomenologia experimental, que utiliza a fenomenologia filosófica para informar e redefinir desenhos experimentais na pesquisa com processos psicológicos básicos. A prerrogativa do modelo front-loaded é o fomento de variações experienciais em seres humanos, por meio de ilusões perceptivas e desempenho cognitivo, sob condições de controle experimental. Ao final, são discutidos os limites da relação entre Fenomenologia e Ciências Cognitivas, apontando a distinção necessária entre Fenomenologia como ciência de fundação e Fenomenologia como pré-ciência experimental aberta ao diálogo interdisciplinar.

Palavras-chave: Cognição; Fenomenologia; Metodologia; Psicologia experimental.

\begin{abstract}
The aim of the paper is to discuss the relationship between Phenomenology and Cognitive Sciences based on what the international literature denominates as Naturalization of Phenomenology. We discuss the research trends in the

\footnotetext{
$\nabla \nabla \nabla$

1 Pontifícia Universidade Católica do Rio Grande do Sul, Faculdade de Psicologia, Programa de Pós-Graduação em Psicologia. Av. Ipiranga, 6681, Prédio 11, Sala 938, 90619-900, Porto Alegre, RS, Brasil. Correspondência para/Correspondence to: T.G. CASTRO. E-mail: <thiago.cast@gmail.com>.

2 Universidade Federal do Rio Grande do Sul, Instituto de Psicologia, Programa de Pós-Graduação em Psicologia. Porto Alegre, RS, Brasil.

Artigo derivado da tese de T.G. CASTRO, intitulada "Percepção e autoconsciência: modelos experimentais na naturalização da fenomenologia". Universidade Federal do Rio Grande do Sul, 2013.

Apoio: Conselho Nacional de Desenvolvimento Científico e Tecnológico (Processo no 140799/2009-5).
} 
field. First, we differentiate the introspective approach methods from the Cognitive Science and Experimental Phenomenology methods. A trend in the naturalization of phenomenology is front-loaded phenomenology, which is characterized as a kind of experimental phenomenology that uses philosophical phenomenology as a tool to inform and refine experimental design settings in research focused on the basic psychological processes. The prerogative of front-loaded model is to promote experiential variation in humans by means of perceptual illusions and cognitive performance, under controlled experimental conditions. Finally, we discuss the limits between Phenomenology and Cognitive Sciences, pointing to the necessary distinction between Phenomenology as a foundational science and Phenomenology as a pre-experimental science open to interdisciplinary dialogue.

Keywords: Cognition; Phenomenology; Methodology; Experimental psychology.

As relações entre a Fenomenologia e as Ciências Cognitivas são analisadas no presente artigo diante das inovações do campo denominado naturalização da fenomenologia. Observou-se na última década uma consolidação da literatura internacional, como pode ser exemplificado pela publicação do "Handbook of Phenomenology and Cognitive Sciences" (Gallagher \& Schmicking, 2010) e pelo fortalecimento do periódico científico dedicado à interface "Phenomenology and the Cognitive Sciences", fundado em 2001. Além dessas iniciativas, a Associação Internacional de Fenomenologia e Ciências Cognitivas (IAPCS), fundada em 2000, mantém suas atividades e encontros anuais para a discussão do tema.

A aproximação das Ciências Cognitivas à teoria fenomenológica clássica passou por renovações nos últimos 10 anos. Anteriormente, prevalecia a crítica fenomenológica à pesquisa experimental. No entanto, o amadurecimento teórico nos últimos anos facilitou a convivência e a cooperação entre Fenomenologia e Ciências Cognitivas. O reconhecimento de interesses comuns entre os dois campos resultou no estudo experimental de processos psicológicos básicos e análise de dados de primeira pessoa. Os resultados são animadores, como mostram os estudos experimentais e fenomenológicos recentes realizados no Brasil, com o "Rubber Hand Illusion" (Botvinick \& Cohen, 1998; DeCastro, 2013) e o "The Alien-hand experiment" (DeCastro \& Gomes, 2011a; Sorensen, 2005).

O artigo está dividido em cinco seções, a saber: 1) Pré-ciência fenomenológica, Fenomenologia Experimental e Introspecção, 2) Ciências Cognitivas, Introspecção e Naturalização da Fenomenologia, 3) Novo projeto conceitual de Fenomenologia Naturalizada, 4) Fenomenologia Naturalizada front-loaded

\section{Pré-ciência fenomenológica, Fenomenologia Experimental e Introspecção}

A noção de pré-ciência fenomenológica, tal como concebida pelo fenomenólogo Carl Stumpf (1848-1936), indica uma ligação afinada entre a Fenomenologia e a experimentação científica (Spiegelberg, 1982). Stumpf é o autor de referência para a "Fenomenologia Experimental como desenvolvida ao longo do século XX". O projeto do fenomenólogo Edmund Husserl (1959-1938), por outro lado, foi apropriado e desenvolvido com maior ênfase em contextos da filosofia hermenêutica e existencial, e das ciências humanas até meados dos anos de 1990 (DeCastro \& Gomes, 2011b).

A noção de Fenomenologia Experimental pode ser situada em três contextos históricos. O primeiro é o contexto das derivações contemporâneas a Husserl nas décadas de 1900 e 1910 para a Psicologia da Gestalt, onde o representante mais associado à Fenomenologia foi o pesquisador Max Wertheimer (Ash, 1998) - associação esta mais ao modo das proposições metodológicas da Fenomenologia de Ewald Hering e Carl Stumpf. Nesse mesmo contexto encontra-se a Escola de Würzburg, encabeçada por Oswald Külpe, que buscou traduzir a ontologia fenomenológica de Husserl para a descrição de mecanismos básicos da percepção em laboratório (Pillsbury, 1911).

O segundo contexto de Fenomenologia Experimental refere-se às apropriações parciais e indiretas da Fenomenologia aos contextos de pesquisa básica experimental. Nesse contexto encontram-se alguns dos alunos de Husserl durante o período na Universidade de Göttingen, como David Katz e Edgar Rubin, mas que se distanciaram aos 
poucos do pensamento fenomenológico, à medida que seus estudos indicaram pouca contribuiç̧ão das mediações autoconscientes e intencionais de sujeitos de pesquisa sobre o desempenho efetivo em tarefas com percepção. Nesses casos a aproximação à Fenomenologia também ocorreu pela via metodológica, como Wertheimer, e obedeceu a uma conexão maior com as indicações do fisiologista Ewald Hering (1834-1918) sobre a utilização de relatos ingênuos na formulação de delineamentos e análise dos dados (Spiegelberg, 1972). O terceiro contexto, e que é alvo de investigação desse artigo, trata da retomada da literatura fenomenológica à luz de achados experimentais nas Ciências Cognitivas, nos últimos 20 anos.

O ponto em comum entre esses três momentos de Fenomenologia Experimental é a proposta de identificação e explicitação das condições que permitem a atuação de uma função perceptiva no comportamento, e não apenas uma descrição dos mecanismos psicofísicos ou do exame de consciência (Sinico, 2010). A ideia da Fenomenologia Experimental é favorecer uma investigação exaustiva das condições de variação da funcionalidade perceptiva. Nessa direção, alguns modelos de experimentação com processos básicos, fundamentados em Fenomenologia, vêm despontando nos últimos anos (Gallagher \& Schmicking, 2010). Além disso, uma linha de argumento metodológico comum aos três contextos históricos indica a valorização da experiência direta de primeira pessoa e as possibilidades de articulação dessa experiência com variáveis indiretas tomadas por instrumentos ou procedimentos objetivos de terceira pessoa (Gallagher \& Zahavi, 2008).

A Fenomenologia é por vezes confundida com os métodos introspectivos característicos da Psicologia alemã da virada do século 19 para o século 20 (Ash, 1998). No tocante aos pensamentos privados e à Fenomenologia Experimental, Gallagher (2010) distingue os propósitos da pesquisa pautada em métodos introspectivos daqueles que fundamentam a pesquisa empírica de base fenomenológica. Segundo o autor, a menção ao termo "introspecção" em contextos experimentais refere-se, em geral, à indicação de acessos reflexivos de segunda ordem, relativos a experiências fenomenais de primeira ordem. Baseando-se nessa definição, Gallagher alega ocorrer uma generalização equivocada sobre o uso da introspecção em todas as formas de pesquisa fenomenológica. Fazer equivalência entre introspecção e acesso reflexivo significaria algo como se todo acesso experiencial passasse por um crivo de julgamento reflexivo antes de se tornar resposta objetiva. Portanto, seria um equívoco tratar a Fenomenologia como sinônimo de método introspectivo.

Críticos da introspecção, como Schooler (2002), indicam que a utilização de qualquer forma de resposta comportamental "experiencial" para a identificação de estímulos em contexto experimental é afetada por vieses subjetivos não desejáveis nesse campo. Para Schooler, mesmo que se tente minimizar o efeito da subjetividade na identificação de estímulos, como pela indicação de respostas através da pressão de botões ou do piscar de olhos, permaneceria ainda uma participação reflexiva, que, segundo o autor, caracteriza a introspecção sobre o julgamento desses estímulos. Há ainda, conforme Schooler (2002), o problema da dissociação temporal entre uma experiência de primeira ordem e a tradução dessa experiência em resposta verbal consciente.

Nesse debate, Gallagher (2010) questiona a ideia de que respostas em primeira pessoa devem necessariamente ser sinônimas de introspecção. Para o pesquisador, na tradição da Fenomenologia, um participante pode relatar sua experiência sem usar a introspecção. Isso porque, para a Fenomenologia, o indivíduo possui uma experiência implícita direta, não introspectiva, e uma autoconsciência pré-reflexiva da experiência. O conhecimento, por exemplo, de enxergar uma luz ou apertar um botão, não é baseado em reflexão ou modificação da atenção por vias introspectivas para a própria experiência. A reação consciente pode ocorrer sem haver introspecção. A experiência tomada em um experimento é em vários casos uma experiência direta, não judicativa, e que define através da ação a recalibração instantânea do curso de uma resposta e os momentos alternados entre experiência da consciência (nem sempre reflexiva) e consciência da 
experiência (esta, sim, judicativa e, portanto, reflexiva).

Para Husserl (1997) a mediação autoconsciente ocorre por monitoramento de uma ação, mas não é pré-requisito para a ação em si tomar seu curso. O curso se define pelas tendências intencionais pré-reflexivas. Assim, fazer uso da experiência em contextos experimentais não é o mesmo que validar relatos de segunda ordem necessariamente orientados por introspecção. Podem ocorrer situações de respostas comportamentais significadas que não passam pelo exercício do crivo reflexivo, como em casos de ambiguidade perceptiva que demandem respostas rápidas. A Fenomenologia Experimental inclui essas modalidades e reconhece, junto às Ciências Cognitivas, a possibilidade de leitura funcional e dinâmica da emissão de respostas sem recorrer necessariamente às análises semânticas da linguagem (Gallagher \& Zahavi, 2008).

\section{Ciências Cognitivas, Introspecção e Naturalização da Fenomenologia}

De acordo com Greenwood (1991), as Ciências Cognitivas, por sua vez, foram por muito tempo desqualificadas no cenário científico da Psicologia, como decorrência de uma associação equivocada do campo ao método introspectivo clássico. O interesse pela cognição e seus modos de acesso às evidências através de experiência consciente levou teorias científicas a um juízo de associação histórica, não justificável, entre as Ciências Cognitivas e o método introspectivo ligado à tradição alemã de pesquisa do final do século XIX. Greenwood ressalta, contudo, que embora ocorra uma associação entre tradições experimentais ligadas à introspecção e as pesquisas cognitivas, tal associação é abstrata, mais vinculada aos interesses de estudo, mas não metodológica. Isso porque o status de evidência nas Ciências Cognitivas não traz referências às impressões de indivíduos em introspecção, mas aos correlatos entre manifestação da experiência consciente e padrões funcionais do aparato biológico. A evidência não se obtém nesse contexto pela confiança no resultado do treino em introspecção como vali-
Para Miller (2003), as Ciências Cognitivas são o produto dos anos 1950, em um tempo em que a Psicologia, a Antropologia e a Linguística estavam se redefinindo, e as Ciências da Computação e as Neurociências eram disciplinas que estavam despontando no cenário científico. Segundo o autor, para a Psicologia poder participar dessa revolução cognitiva, era necessário restaurar uma respeitabilidade científica ao tema da cognição, e nesse sentido um divórcio com o eliminativismo behaviorista era iminente. A nova proposição científica precisava de novas teorias, mais colaborativas entre campos do saber, e mais pautadas no funcionamento conjunto entre observação de comportamento e biologia do comportamento.

Conforme indicado por McClelland (2010), as Ciências Cognitivas passaram por três demarcações históricas, caracterizadas, entre as décadas de 1950 até meados dos anos 1990, pelo estudo dos processos mentais explicitados metaforicamente por abstrações conceituais. Primeiramente, com o cognitivismo, dominante entre 1950 e 1980, criou-se a metáfora da mente como um computador digital. Depois, com o conexionismo, dominante na década de 1980, a mente era entendida como uma rede neural. Mais recentemente, com a teoria do dinamicismo incorporado, que teve grande impacto na década de 1990, a metáfora da mente foi representada pela tese dos sistemas dinâmicos cognitivos incorporados ao mundo.

No panorama conceitual das teorias dos sistemas dinâmicos e do conexionismo, McClelland (2010) avalia que a noção de "emergência" foi a responsável pelo início da mudança nas explicações sobre a cognição. Desse modo, passou-se de uma fase em que os processamentos cognitivos centravam-se em sistemas de execução por comando central e efeitos de cascata em subsistemas (cognitivismo computacional), ao entendimento da cognição como um processo emergente em redes interconectadas. O conceito de emergência é antigo, mas demorou a aparecer nas Ciências Cognitivas.

De acordo com Lewes (1875/2005), o conceito de emergência contrasta com o conceito de resultado. Segundo o autor, em paradigmas pautados por resultado, cada resultado é a soma ou a 
diferença de forças em cooperação. Seria diferente com a emergência, onde ocorre uma cooperação não concorrente entre as forças. O emergente é diferente de seus componentes, na medida em que tais elementos são incomensuráveis, e o emergente não pode ser reduzido à soma ou à diferença de seus componentes. Observa-se aqui clara similaridade entre emergência e o princípio de Gestalten.

Ainda que ocorra um desenvolvimento histórico não paralelo entre o conexionismo e a teoria dos sistemas dinâmicos, Thelen e Bates (2003) entendem que as teorias não competem. Os autores observam uma base comum entres as propostas, qual seja a relação entre informações externas ambientais e o processamento mental dessas informações. As informações externas são críticas para o desempenho comportamental em ambas as teorias, porém, mesmo que alterações no ambiente modifiquem o comportamento, não é o ambiente o ponto final dessas modificações, mas sim a estrutura mental. No conexionismo, a explicação dessas modificações mentais é mais carregada na ênfase das representações mentais internas sobre os comportamentos futuros, enquanto na teoria dos sistemas dinâmicos a ênfase recai sobre a ação motora exploratória e a adaptação direta do organismo ao ambiente.

Thelen e Bates (2003) indicam ainda que a teoria dos sistemas dinâmicos iniciou no campo experimental dos estudos em percepção e ação, e tem entre seus representantes mais distantes o psicólogo James Gibson (1904-1979). O impacto mais forte da teoria é notado no desenvolvimento sensório-motor em crianças. Já o conexionismo emergiu da modelação computacional e tem seu foco no mapeamento interno do cérebro e seus conteúdos representacionais. Segundo os autores, as diferenças entre as teorias recaem mais nos métodos empregados nos estudos e nas perguntas de pesquisa, mas existem semelhanças no entendimento da cognição e na abertura à influência de outras ciências, como neurobiologia, física, matemática e ciências da computação.

A literatura dos sistemas dinâmicos, especialmente, iniciou na década de 1990 uma incursão na literatura fenomenológica para reforçar o entendimento emergentista nas Ciências Cognitivas. O texto "Mente incorporada", de Varela, Thompson e Rosch (1991), demarcou uma primeira aproximação tímida com a Fenomenologia. No livro, os autores discutiram a Fenomenologia de Husserl brevemente, criticando-a pelo isolacionismo do ego na constituição da experiência significada consciente (Varela et al., 1991). Posteriormente, Varela (1996) desenvolveu melhor sua proposta, a qual nomeou Neurofenomenologia, reanalisando a Fenomenologia de Husserl e situando os temas do filósofo em sua solução metodológica. A Neurofenomenologia de Varela procurava integrar três elementos: a) análise fenomenológica da experiência (qualidades); b) a teoria dos sistemas dinâmicos; e c) a experimentação empírica em sistemas biológicos (Gallagher, 2010). O objetivo de Varela com isso seria superar a lacuna explicativa entre metáforas mentais e dinâmicas neurofuncionais da consciência. Essa lacuna de explicações entre metáfora e matéria da consciência recebeu o nome de "Hard Problem", inicialmente pelo filósofo Chalmers (1995) e em seguimento por cientistas da consciência, como Varela.

Esse primeiro momento de aproximação de cientistas cognitivos à Fenomenologia, no texto "Mente incorporada" (1991), foi posteriormente avaliado por Thompson (2007) como um acesso não confiável à literatura clássica fenomenológica. Conforme o autor, a análise crítica feita ao texto husserliano em 1991 foi muito precipitada, faltando conhecimento suficiente e aprofundado das nuances do texto analisado. Segundo Thompson (2007), os autores haviam sido levados a uma interpretação equivocada da Fenomenologia de Husserl pela interpretação do filósofo norte americano Hubert Dreyfus. Thompson e seus colegas foram influenciados pelos comentários de Dreyfus (1982), que considerava Husserl um cognitivista ao modo protocomputacionalista - isto é, um autor afinado com versões computacionais de processamento da informação sobre o funcionamento da mente. Tal versão de classificação, amplamente rejeitada nos dias de hoje, foi revisada e negada primeiramente por Varela (Petitot, Varela, Pachoud, \& Roy, 1999) e depois por Thompson (2007). 


\section{Novo projeto conceitual de Fenomenologia Naturalizada}

A renovação da inserção da Fenomenologia nas Ciências Cognitivas pelo grupo de Varela, ao final da década de 1990, deixou ainda dúvidas sobre a possibilidade de uma naturalização da Fenomenologia. Mesmo que os autores tenham revisado sua interpretação do texto husserliano, conferindo à Fenomenologia lugar de destaque para uma nova orientação das Ciências Cognitivas, críticas sobre essa via de naturalização foram levantadas (Bayne, 2004). As críticas apontaram, em geral, uma suposta fragilidade e superficialidade do projeto, ao incorporar a Fenomenologia sem tratar cuidadosamente das diferenças entre as tradições teóricas. De fato, Petitot et al. (1999), expoentes da Neurofenomenologia, deixaram claro seu objetivo de reorientar a Fenomenologia de Husserl, indicando a necessidade de uma mudança radical nessa literatura. Os autores discorreram sobre um "movimento recíproco" de reforçar a pesquisa cognitiva com a ajuda da Fenomenologia de Husserl e uma necessidade de transformação da Fenomenologia em suas raízes (Petitot et al., 1999). Contudo, a ideia de naturalização nesse projeto expôs uma tentativa ingênua de fusão forçada por reciprocidade entre tradições filosóficas muito distintas.

Mesmo indicando uma boa iniciativa de incorporar a Fenomenologia em um cenário científico, faltou ao projeto de Varela o entendimento de uma independência entre filosofia e apropriações empíricas. Conforme Schmicking (2010), a Fenomenologia, quando naturalizada por inserção direta no trabalho científico, pode correr o risco de se tornar alheia aos pressupostos das ciências empíricas. E, para a Fenomenologia, tornar-se alheia às lógicas que compõem uma investigação seria no mínimo um contrassenso ou, então, um erro de definição do trabalho fenomenológico. Tais pressupostos não podem ser refletidos dentro do próprio âmbito do fazer científico, por isso a impossibilidade de incluir a Fenomenologia, que é uma filosofia, como um instrumental ou remédio metodológico, como queria a Neurofenomenologia.

Nesse período que vai de meados dos anos 400 crítica à inclusão de protocolos subjetivos na pesquisa neurocientífica. A chamada Heterofenomenologia, proposta por Dennett (2003), tratou de definir o estudo da consciência humana como um campo da objetividade, em terceira pessoa, pela busca da neutralidade e afastamento das impressões pessoais de participantes dos estudos, impressões estas chamadas pelo filósofo como ficções. Dennett denominou o campo como Heterofenomenologia, pois entendeu que a Fenomenologia deve ser a descrição de outra pessoa olhando de fora o que se passa objetivamente na experiência de um indivíduo em condição experimental. De acordo com Van de Laar (2008), ainda que sejam notórias as diferenças de pressupostos e metodologia entre a Neurofenomenologia e a Heterofenomenologia, na prática é ainda difícil fazer uma escolha entre ambas para o estudo da consciência. Talvez essa dificuldade seja decorrente das incertezas sobre a efetividade de um ou outro procedimento. De um lado, o uso de relatos subjetivos pode ser uma abertura a novas formas de interpretação dos dados neurofuncionais, mas, de outro, corre-se o risco de promover equivalência entre processos verbais e dinâmicas neurofuncionais dissociadas, como atenta a Heterofenomenologia.

Recentemente, Zahavi (2010) fez uma análise sobre dois projetos que buscaram a naturalização da Fenomenologia nos últimos 15 anos. Para o autor, existem dois entendimentos hegemônicos paralelos, porém distintos, de naturalização da Fenomenologia clássica. O primeiro incorre na compreensão de que a Fenomenologia faria parte, ou ao menos seria uma extensão, das ciências naturais - algo como uma nova corrente analítica de explicação dos dados naturais. Conforme o autor, essa proposta cometeria o erro de promover uma equivalência entre a metodologia científica comprometida com a empiria, segundo sua própria definição, e a reflexão filosófica fenomenológica que problematiza aspectos transcendentais da aparência do empírico. Nesse caso, a Fenomenologia seria um método acoplado ao sistema explicativo, partindo de uma base homogênea de compreensão do dado natural a ser investigado. Tal viés de naturalização refere-se claramente à proposta neurofenomenológica encabeçada por Varela nos anos de 1990 
e continuada por Antoine Lutz (Lutz, Lachaux, Martinerie, \& Varela, 2002) e Michel Le Van Quyen (Le Van Quyen, Martinerie, Navarro, Baulac, \& Varela, 2001; Le Van Quyen \& Petitmengin, 2002) nos anos 2000.

Segundo Zahavi (2010), essa primeira fórmula de naturalização distorceria o real significado do projeto fenomenológico, mesmo que recorrendo à literatura clássica da Fenomenologia de Husserl. Zahavi indica que esse modelo de naturalização produz normatividade e rigidez às idealidades da consciência, tornando um processo de significação estendido no tempo em algo estático. Além disso, coloca a própria consciência como um objeto no mundo, o que rompe radicalmente com qualquer argumento fenomenológico. Nesse sentido, o problema vislumbrado na primeira geração da naturalização seria relegar a segundo plano, ou mesmo ignorar, a distinção entre aspectos transcendentais da consciência e o seu estudo como objeto localizável em estruturas cerebrais.

O segundo entendimento de naturalização, conforme Zahavi (2010), apresenta uma proposta mais modesta, na qual a Fenomenologia deve ser resgatada para um intercâmbio produtivo sobre os fundamentos metodológicos e epistemológicos que sustentam os achados das ciências empíricas. Nessa formulação, a reflexão fenomenológica deve servir como anteparo para a discussão das lógicas inferenciais nas análises empíricas e, inclusive, ser desafiada em suas análises pelos refinamentos experimentais atuais. O objetivo desse empreendimento de naturalização é validar settings experimentais por variações experienciais, e não por confirmação de hipótese. A noção de Fenomenologia front-loaded, apresentada inicialmente por Gallagher (2003) e redefinida no decorrer da última década (Gallagher, 2010; Gallagher \& Sorensen, 2006; Gallagher \& Zahavi, 2008), é representante da segunda geração de naturalização da fenomenologia.

\section{Fenomenologia Naturalizada front-loaded e Pesquisa Experimental}

Segundo Gallagher e Sorensen (2006), os usos da Fenomenologia nas Ciências Cognitivas se concentram no campo tradicional da Fenomenologia Experimental e contemplam duas modalidades de condução de pesquisa. A primeira forma refere-se ao treino de resposta dos participantes no contexto experimental e no método fenomenológico. Seus relatos de experiência auxiliam qualitativamente a redefinir, por exemplo, os critérios de avaliação de protocolos neurofuncionais (Lutz et al., 2002). Essa modalidade está associada à Neurofenomenologia. A segunda perspectiva de Fenomenologia experimental contemporânea, denominada front-loaded, não envolve o treino dos participantes no método fenomenológico, solicitando apenas ocasionalmente relatos de experiência. Baseia-se, sobretudo, na definição do enquadramento interpretativo dos resultados da pesquisa empírica, a partir de um contraste dos achados com conceitos advindos da literatura fenomenológica. Tais conceitos podem tanto informar o desenho metodológico quanto rediscutir definições operacionais correntes de processos psicológicos básicos na literatura.

Gallagher (2010) rediscutiu a relação entre Fenomenologia e pesquisa experimental, citando possibilidades de utilização de dados de primeira pessoa em delineamentos experimentais. O autor caracterizou os usos metodológicos da literatura fenomenológica na Neurofenomenologia e na Fenomenologia front-loaded. No caso da Neurofenomenologia, Gallagher avalia que a reunião entre a Fenomenologia de Husserl e as Ciências Cognitivas gera um tipo de estudo dos atos pré-conscientes em experimentos que incluem a descrição experiencial consciente apenas após o desempenho de determinadas tarefas (Lutz \& Thompson, 2003). Os experimentos consistem na proposição de uma tarefa experiencial e motora, passível de ser descrita conforme treino descritivo estipulado pelos pesquisadores, com o objetivo de verificar a associação entre a descrição e o desempenho corporal motor. O treino dos participantes envolve principalmente a prática de suspensão de julgamentos valorativos na ocasião da descrição da experiência - redução fenomenológica (Depraz, Varela, \& Vermersch, 2006). Há, contudo, nesse cenário um delay temporal importante entre ação e julgamento, o que 
abre brechas aos críticos das pesquisas baseadas em relatos de primeira pessoa (Schooler, 2002; Dennet, 2003).

Por outro lado, a Fenomenologia frontloaded não possui pretensões de responder às lacunas entre consciência e dinâmicas neurofuncionais. Seu principal objetivo é definir pontos de contribuição de insights fenomenológicos para a montagem de settings experimentais que propiciem variações experienciais. Nesse sentido, almeja uma troca dialética entre recursos de informação metodológica e operacional dos experimentos e caracterizações de possíveis observações da experiência em sua variabilidade ao longo do tempo (Gallagher, 2010). Os usos ocorrem mais na etapa dos estudos pilotos e indicam uma composição de settings não pautada em achados anteriores da literatura, mas na modificação de settings para entender as eventuais modificações da experiência. Ou seja, o objetivo aí não é a corroboração, mas a variação experiencial. Tem por finalidade clarificar as distinções fenomenológicas e insights das experiências no contexto experimental, para contribuir no design experimental e na interpretação dos achados.

\section{Discussão}

Ainda que Husserl considere a necessidade das ciências empíricas buscarem um tipo de entendimento que apenas a Fenomenologia transcendental poderia alcançar, a realização de tal empreendimento só poderia ocorrer no contexto de um discurso interdisciplinar. O recuo histórico à Fenomenologia desperta curiosidades entre cientistas cognitivos sobre as possibilidades e viabilidades de uma releitura do empírico em pesquisas. Fornece também pistas sobre variações metodológicas para o alcance da experiência consciente em primeira pessoa ou combinação de dados de primeira e terceira pessoa. Contudo, a literatura da naturalização da Fenomenologia vem afirmando uma via moderada de apropriação da Fenomenologia clássica para as investigações contemporâneas. Os cientistas cognitivos não parecem estar à procura de uma teoria de fundação para sua compreensão de consciência, tampouco a ciência em geral está longe de indicar uma via única para uma teoria de fundação.

Aliás, a noção de que um sistema filosófico pode definir o direcionamento de um campo de investigações empíricas parece ter sido abandonada já no início do século XX. O avanço da pesquisa experimental demonstrou que teorias de base, como a Gestalt ou o Behaviorismo, não conseguem explicar perfeitamente toda a variabilidade do funcionamento do organismo em diferentes situações. Uma evidência dessa incompletude teórica é a consolidação de eventos científicos e departamentos de pesquisa em universidades europeias e norte-americanas, nos últimos 15 anos, para o estudo integrado e interdisciplinar das funções cognitivas. O trabalho usual em Psicologia, que costumava buscar a corroboração de teorias específicas pela interpretação de achados empíricos, vem cedendo espaço para a contribuição entre teorias na informação fidedigna do funcionamento da cognição. Ou seja, observa-se uma clara inversão de pressupostos. Onde antes se mantinha uma lógica de acoplamento teórico-empírico, com a teoria resguardando lugar de destaque na investigação, tem-se agora a utilização das ideias (teorias) para a geração de novas formas de produção empírica e posterior ilustração das incompletudes dos sistemas teóricos. A teoria tem, nesse sentido, dupla função: a) primeiro, de elucidação descritiva e tangível do funcionamento cognitivo; b) segundo, de geração de novas perguntas de pesquisa para a criação de variações experimentais e experienciais. Em suma, teorias de fundação ou ciências primeiras, em linguagem husserliana, parecem ter perdido força com o desenvolvimento de versões colaborativas de ciência.

Nesse contexto de intercâmbio, as utilizações da Fenomenologia nas Ciências Cognitivas não se limitam à refundação da direção das pesquisas empíricas ou à modificação da interpretação do texto clássico fenomenológico. Com os avanços tecnológicos no campo de integração multissensorial para o estudo da percepção, por exemplo, várias perguntas sobre a unificação da experiência sensorial na consciência começaram a despertar o interesse dos cientistas. Aqui, a retomada da Fenomenologia mostrou-se mais como um recurso de observação 
na história da filosofia, para entender modelos conceituais do fluxo das experiências na consciência, do que propriamente uma tentativa de refundação das ciências empíricas.

É natural, nesse sentido, indicar as diferenças entre as noções de pré-ciência de Carl Stumpf e ciência primeira de Husserl. O entendimento de pré-ciência é menos fundacionista e atenta para a importância de se ter claro um espectro de evidências que vão além da explicação causal de fenômenos naturais para a natureza da experiência consciente. Stumpf via a necessidade de submeter a Fenomenologia a um exercício profícuo de diálogo com as ciências empíricas, sem o qual sua função não faria sentido. Por outro lado, uma versão mais fundacional sobre a experiência da consciência resultará em uma Fenomenologia transcendental, que entende o estudo experimental de terceira pessoa como uma ferramenta inapropriada para apreender as manifestações da subjetividade.

Diante do paradoxo entre as negativas sobre a viabilidade de uma Fenomenologia naturalizada e o andamento crescente de pesquisas nesse campo, a melhor sugestão é a de uma versão provisória de Fenomenologia naturalizada. Nessa direção, seria mais apropriado abandonar as reivindicações de ciência primeira e buscar validar ferramentas de primeira pessoa e combiná-las com ferramentas de segunda e terceira pessoas. Buscar reconhecer na Fenomenologia a relevância dos dados e teorias científicas para si, assim como a receptividade de seu tema de estudo para as ciências empíricas. Essa seria a versão de trabalho de Fenomenologia naturalizada no campo científico atual, distante de um contexto de manutenção de status ou vinculação estrita ao antinaturalismo.

\section{Referências}

Ash, M. G. (1998). Gestalt psychology in german culture, 1890-1967: Holism and the quest for objectivity. New York: Cambridge University Press.

Bayne, T. (2004). Closing the gap? Some questions for neurophenomenology. Phenomenology and the Cognitive Sciences, 3(4), 349-364.

Botvinick, M., \& Cohen, J. (1998). Rubber hands 'feel' touch that eyes see. Nature, 391(6669), 756.
Chalmers, D. (1995). Facing up to the problem of consciousness. Journal of Consciousness Studies, 2(3), 200-219.

DeCastro, T. G. (2013). Percepção e autoconsciência: modelos experimentais na naturalização da fenomenologia (Tese de doutorado não-publicada). Universidade Federal do Rio Grande do Sul.

DeCastro, T. G., \& Gomes, W. B. (2011a). Autoconsciência e ambiguidade perceptual cinestésica: experimento fenomenológico. Psicologia em Estudo, 16(2), 279-287.

DeCastro, T. G., \& Gomes, W. B. (2011b). Movimento fenomenológico: controvérsias e perspectivas na pesquisa psicológica. Psicologia: Teoria e Pesquisa, 27(2), 233-240.

Dennett, D. (2003). Who's on first? Heterophenomenology explained. Journal of Consciousness Studies, 10(9-10), 19-30.

Depraz, N., Varela, F. J., \& Vermersch, P. (2006). A redução à prova da experiência. Arquivos Brasileiros de Psicologia, 58(1), 75-86.

Dreyfus, H. (1982). Introduction. In H. Dreyfus \& H. Harrison (Eds.), Husserl, intentionality and cognitive sciences. Cambridge, MA: MIT Press.

Gallagher, S. (2003). Phenomenology and experimental design toward a phenomenologically enlightened experimental science. Journal of Consciousness Studies, 10(9-10), 85-99.

Gallagher, S. (2010). Phenomenology and non-reductionist cognitive science. In S. Gallagher \& D. Schmicking (Eds.), Handbook of phenomenology and cognitive sciences (pp.21-34). New York: Springer.

Gallagher, S., \& Sorensen, J.P. (2006). Experimenting with phenomenology. Consciousness and Cognition, 15(1), 119-134.

Gallagher, S., \& Zahavi, D. (2008). The phenomenological mind. London: Routledge.

Gallagher, S., \& Schmicking, D. (2010). Handbook of Phenomenology and Cognitive Sciences. New York: Springer.

Greenwood, J. D. (1991). The future of folk psychology: Intentionality and cognitive science. Canadá: Cambridge University Press.

Husserl, E. (1997). Thing and space: Lectures of 1907. Netherlands: Kluwer Academic Publisher.

Le Van Quyen, M., Martinerie, J., Navarro, V., Baulac, M., \& Varela, F. J. (2001). Characterizing the neurodynamical changes prior to seizures. Journal of Clinical Neurophysiology, 18(3), 191-208.

Le Van Quyen, M., \& Petitmengin, C. (2002). Neuronal dynamics and conscious experience: An example of reciprocal causation before epileptic seizures. Phenomenology and the Cognitive Sciences, 1(2), 169-180. 
Lewes, G. H. (2005). Problems of life and mind. First Series, Michigan Series: Scholarly Publishing Office. Ann Arbor, MI: University of Michigan Library. (Original published in 1875).

Lutz, A., Lachaux, J.P., Martinerie, J., \& Varela, F. (2002). Guiding the study of brain dynamics by using firstperson data: Synchrony patterns correlate with ongoing conscious states during a simple visual task. Proceedings of the National Academy of Sciences, 99(3), 1586-1591.

Lutz, A., \& Thompson, E. (2003). Neurophenomenology: Integrating subjective experience and brain dynamics in the neuroscience of consciousness. Journal of Consciousness Studies, 10(9/10), 31-52.

McClelland, J. L. (2010). Emergence in cognitive science. Topics in Cognitive Science, 2(4), 751-770.

Miller, G. A. (2003). The cognitive revolution: A historical perspective. Trends in Cognitive Sciences, 7(3), 141-144.

Petitot, J., Varela, F., Pachoud, B., \& Roy, J. M. (1999). Naturalizing Phenomenology: Issues in contemporary phenomenology and cognitive sciences. Stanford, CA: Stanford University Press.

Pillsbury, W. B. (1911). The essentials of psychology. New York: McMillan.

Schmicking, D. (2010). A toolbox of phenomenological methods. In S. Gallagher \& D. Schmicking (Eds.), Handbook of phenomenology and cognitive sciences (pp.35-56). New York: Springer.

Schooler, J. W. (2002). Re-presenting consciousness: Dissociations between experience and metaconsciousness. Trends in Cognitive Sciences, 6(8), 339-344.

Sinico, M. (2010). Explanation in experimental phenomenology: The nomic, deterministic and modal domain. Gestalt Theory, 32(4), 293-306.
Sorensen, J. B. (2005). The Alien-hand experiment. Phenomenology and the Cognitive Sciences, 4(1), 73-90.

Spiegelberg, H. (1972). Phenomenology in psychology and psychiatry: A historical introduction. Evanston, IL: Northwestern University Press.

Spiegelberg, H. (1982). The phenomenological movement: A historical introduction. Boston, MA: Martinus Nihjhoff.

Thelen, E., \& Bates, E. (2003). Connectionism and dynamic systems: Are they really different? Developmental Science, 6(4), 378-391.

Thompson, E. (2007). Mind in life: Biology, phenomenology, and the sciences of mind. Cambridge, MA: Harvard University Press.

Van De Laar, T. (2008). Mind the methodology: Comparing heterophenomenology and neurophenomenology as methodologies for the scientific study of consciousness. Theory \& Psychology, 18(3), 365-379.

Varela, F. J. (1996). Neurophenomenology: A methodological remedy to the hard problem. Journal of Consciousness Studies, 3(4), 330-349.

Varela, F. J., Thompson, E., \& Rosch, E. (1991). The embodied mind. Cambridge, MA: MIT Press.

Zahavi, D. (2010). Naturalized phenomenology. In S. Gallagher \& D. Schmicking (Eds.), Handbook of phenomenology and cognitive science (pp.3-19). New York: Springer.

Received: June 3, 2013

Final version: May 23, 2014

Approved: July 11, 2014 